\title{
Electrical Hazards Posed by Graphite Fibers
}

\author{
George S. Springer, Safer A. Mashtizadeh and \\ ROBERT B. KELLER
}

Department of Mechanical Engineering

The University of Michigan,

Ann Arbor, Michigan 48109

(Received April 9, 1979)

\begin{abstract}
The direct electrical resistance and the electrical contact resistance of graphite fibers released from both burned and unburned Fiberite T300/1034 composites were measured. The voltage at which arcing occurs due to fibers settling on electrical conductors was also determined. On the basis of results obtained, an assessment was made of the hazards posed by graphite fibers to electrical equipment.
\end{abstract}

\section{INTRODUCTION}

\begin{abstract}
Wing to THEIr favorable performance characteristics composite materials have been gaining wide use in commercial, space, and military applications. There is some concern now about the use of graphite fiber reinforced composite materials, due to hazards posed by fibers when they are accidentally released into the atmosphere. Such fibers may interfere with or damage unprotected electrical equipment by causing resistive loading, temporary short circuits, or arcing. These detrimental effects are enhanced by the light weight of the fibers, a factor which facilitates their transport through the atmosphere.

That graphite fibers released from composite materials present a hazard to electrical equipment has been recognized, and the need for accident prevention has been publicized [1-5]. However, very few data are available in the open literature which describe quantitively the effects of graphite fibers on electrical equipment $[4,5]$. Therefore, the objective of this investigation was to obtain data indicating the magnitudes of the parameters at which graphite fibers would damage electrical circuits and equipment.
\end{abstract}




\section{EXPERIMENTAL}

The following three parameters were measured: 1) the direct resistance of the fibers, 2) the contact resistance of the fibers, and 3) the d.c. voltage at which arcing occurs at the fiber-conductor interface.

The direct resistance of the fibers was measured by enclosing tightly the two ends of the fibers between copper clips (Figure 1). The resistance was measured with a Hewlett Packard ohm meter. The contact resistance was measured by placing the fibers on top of two copper conductors. The resistance of the circuit was also measured with the Hewlett Packard ohm meter (Figure 1).

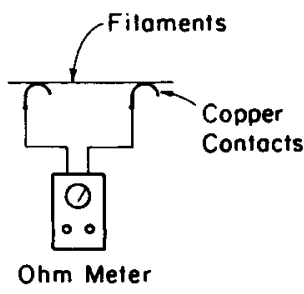

Type of Contocts:

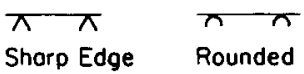

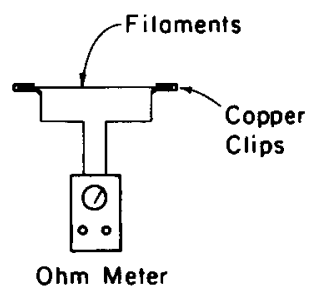

Flot

Figure 1. Schematic of apparatus used in measuring the contact resistance (left) and the direct resistance (right) of fiber bundles. Lower figure shows the types of contacts used in measuring the contact resistance and the voltage at arcing.

The voltage required to produce arcing was determined as follows. The fibers were placed on top of two copper conductors connected to a power supply (Figure 2 ). The voltage drop across the fibers was measured by a Simpson volt meter. The current through the fibers was monitored by placing a low resistance shunt in series with the fibers and by transmitting the voltage drop across this shunt to the differential amplifier of a Tektronix oscilloscope. By using a-c coupling, the sudden increase of current, which is associated with the onset of arcing, was displayed on the oscilloscope.

"Burned" fibers were obtained by igniting the composite with a Bunsen burner. The material was burned until all resin was burned away and the fibers peeled off readily. "Unburned" fibers were removed directly from the composite by cutting out a slice containing both resin and fiber. 


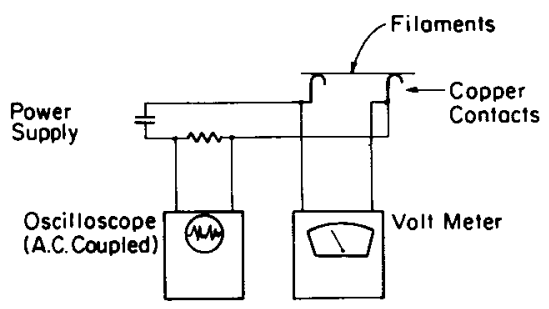

Figure 2. Schematic of apparatus used in measuring the voltage at which arcing occurs.

\section{RESULTS}

The problem at hand is to determine under what circumstances electrical circuits will be affected by the presence of fibers on uninsulated portions of circuit conductors and components. The fibers may cause short circuits resulting in arcing, spurious signals, and resistive loading. Arcing is primarily a function of voltage; resistive loading and the propagation of spurious signals depend primarily on the fiber resistance across the conductors. Hence, the two parameters of major interest are the voltage at which arcing occurs and the resistance across the fibers. The objective of this investigation was to measure these parameters under conditions which simulate practical engineering conditions, and to determine their dependence upon fiber length, number of fibers in the bundle, and the type of electrical contact.

Fiberite T300/1034 composites were used throughout the tests. The specimens were obtained from $0.66 \mathrm{~m} \times 0.66 \mathrm{~m}$ autoclave cured panels which were fabricated from $30.5 \mathrm{~cm}$ (12 in.) prepreg (Fiberite Corp.) using standard lay-up and vacuum bagging procedures. The cure cycle used in manufacturing the panels was described in $[6]$.

The fiber and conductor surfaces were not cleaned prior to or during the tests. It is recognized that the condition of the contact surfaces may also influence "voltage at arcing" and fiber resistance. The surface conditions employed in the present tests are believed to have simulated conditions which would be encountered in actual, real life situations.

Tests were performed with five different fiber lengths $(2.5,5.0,7.5,10.0$, and $12.5 \mathrm{~mm}$ ), with five different fiber bundles (composed of $5,10,15,20$ and 50 fibers per bundle) and with four different pairs of electrical conductors. One pair of conductors was a sharp edge, one was a flat edge and two were circular with 3.2 and $1.6 \mathrm{~mm}$ diameters (Figure 1). The data showed that the resistance was insensitive to the shape of the conductors. Differences in contact resistance and "voltage at arcing" using different conductors were within the spread of the data. Hence, in the presentation of the data, the type of conductor is not identified separately.

The resistance of both burned and unburned fibers are shown in Figure 3. As can be seen, both the direct and the contact resistances of the unburned fibers are 

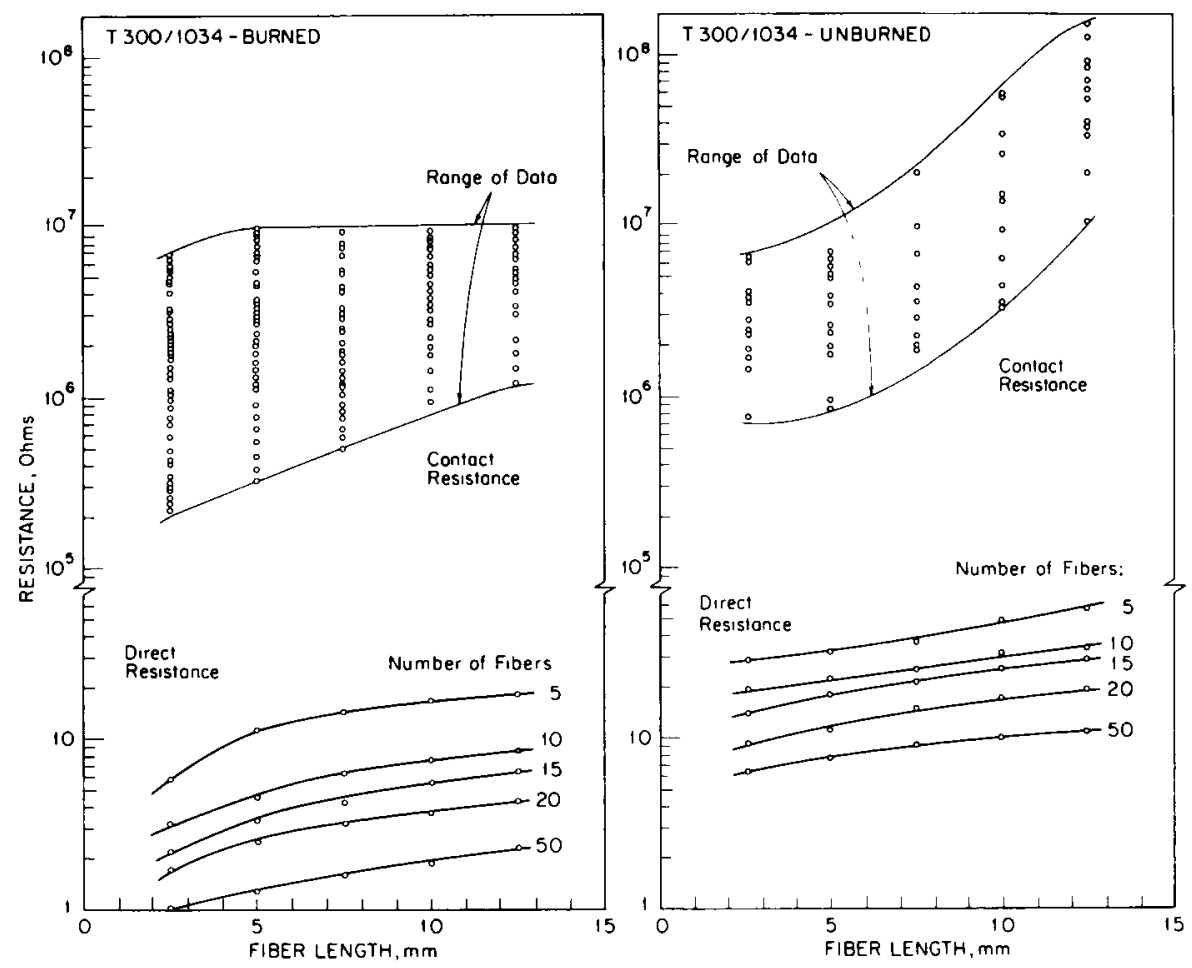

Figure 3. The direct resistance (bottom) and the contact resistance (top) of fiber bundles removed from burned (left) and unburned (right) composite.

somewhat higher than the direct and contact resistances of the burned fibers. Furthermore, the contact resistance is about five orders of magnitude higher than the direct resistance. Here we are mostly concerned with the contact resistance since contact resistance occurs when fibers settle on equipment. The contact resistance is in the range of 0.25 to 10 megohms for burned fibers and 0.7 to 200 megohms for unburned fibers. Within the spread of the data, the contact resistance appears to be insensitive to the number of fibers and depends only on the fiber length.

The foregoing results suggest that contact resistance will not cause significant adverse effects as long as the circuit impedance level is lower than megohms. The input impedance of many circuits is in the megohm range. For such circuits the fibers would produce serious performance reduction. Only those circuits which have impedances in the range of 10 to 1000 ohms would probably not be affected by the fibers provided, of course, that the voltage level is not high enough to produce arcing.

The voltage at which arcing occurs between the fibers and the conductor is 
shown in Figures 4 and 5. The results in Figure 4 indicate that the voltage is influenced by the number of fibers but not by the fiber length. For fibers removed from burned composites arcing occurs at relatively low voltages (5 to 10 volts). For unburned fibers (i.e., for fibers embedded in unburned resin) the arcing voltage was higher than for unburned fibers but was still less than 20 volts (Figure 5).

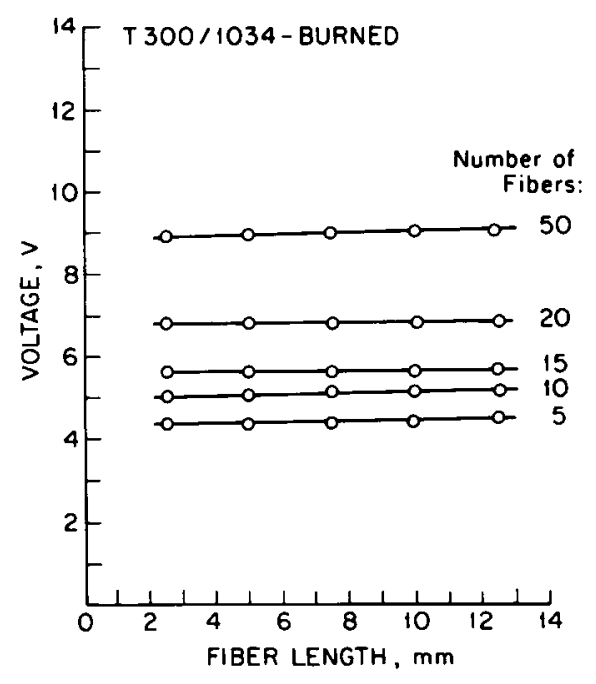

Figure 4. Voltage at which arcing first occurs at the filament conductor interface. Fibers removed from burned composites. $\mathrm{O}$ average of the data, ---- fit to data.

The values shown in Figure 4 are the lowest voltages at which arcing occurs. As the voltage is increased beyond the minimum value arcing becomes more pronounced and is often accompanied by a "bouncing" movement of the fibers. Arcing is intermittent. The following is a plausible explanation for this phenomenon. Arcing occurs when the voltage "punches through" the fiber surface layer. As the fibers move, new, undamaged fiber surface comes into contact with the conductor. Arcing reoccurs only when this fresh layer is "punched through."

It is noted that when the voltage becomes sufficiently high the fibers may also ignite and burn.

The data in Figures 3 and 5 show that both the fiber resistance and the voltage at arcing are higher for fibers coated with resin than for fibers without the resin coating. These data thus bear out the observation noted previously that fiber resistance and voltage at arcing depend upon the fiber surface. This suggests that protective coatings might provide possible means of minimizing problems created by graphite fibers. 


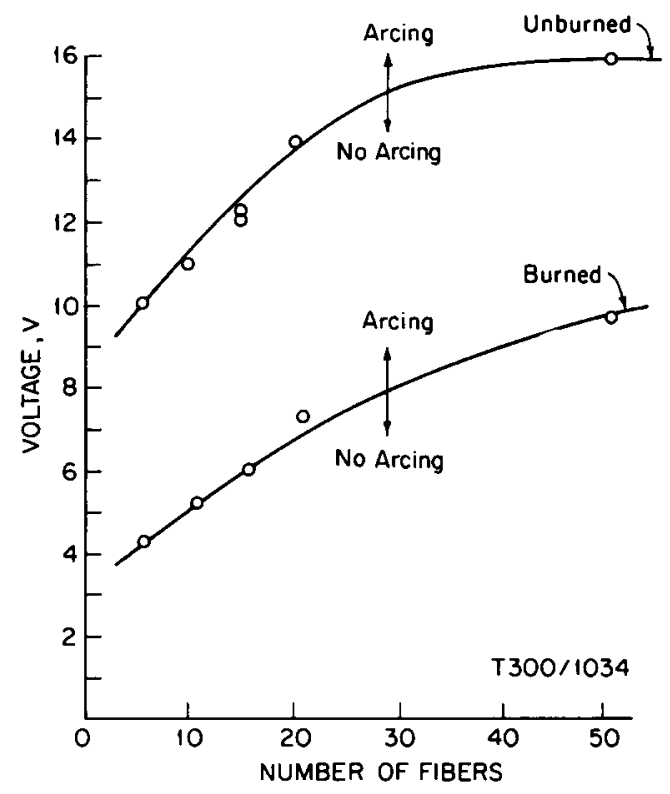

Figure 5. Voltage at which arcing first occurs at the filament conductor interface. Fibers removed both from burned and unburned composites. $\mathrm{O}$ average of the data, fit to data.

\section{SUMMARY}

The electrical hazards posed by graphite fibers released from Fiberite T300/1034 composites were evaluated at conditions simulating practical situations. The data obtained indicated the following:

1) Although the direct resistance of the fibers is low (less than $20 \mathrm{ohms}$ ), the contact resistance of 0.25 to $12.5 \mathrm{~mm}$ long fibers is in the range of 0.25 to 10 megohms for fibers removed from burned composites and is in the range of 0.7 to 200 megohms for fibers removed from unburned composites.

2) Fibers removed from burned composites cause arcing at voltages as low as 4 to 10 volts. Fibers removed from unburned composites cause arcing at slightly higher voltages (10 to 20 volts).

\section{ACKNOWLEDGMENT}

This work was supported by NASA Grant NSG-2333 and by the United States Air Force Materials Laboratory. 


\section{REFERENCES}

1. United States Department of Commerce News Release, January 20, 1978.

2. "A Report of Observed Effects on Electrical Systems of Airborne Carbon/Graphite Fibers," NASA TM 78652 (1978).

3. J. H. McFerrin and O. C. Trulson, "Electrical Equipment Protection and Waste Disposal Practices - Carbon Fiber Manufacture," SAE paper 790034 (1979).

4. I. Taback, "Vulnerability" in Carbon Fiber Risk Analysis, NASA Conference Publication 2074 (1978), p. 109.

5. "Carbon Fiber Electrical Resistance Modification - Its Relationship to Electrical Equipment Malfunctions," Final Report of the Office of Naval Research Carbon Fiber Study Group, Office of Naval Research, Metallurgy and Ceramic Program (1978).

6. C. H. Shen and G. S. Springer, "Effects of Moisture and Temperature on The Tensile Strength of Composite Materials," J. Composite Materials, Vol. 11 (1977), p. 2. 